\title{
SHARP FRONTS DUE TO DIFFUSION AND VISCOELASTIC RELAXATION IN POLYMERS*
}

\author{
DONALD S. COHEN† AND ANDREW B. WHITE, JR. $\ddagger$
}

\begin{abstract}
A model for sharp fronts in glassy polymers is derived and analyzed. The major effect of a diffusing penetrant on the polymer entanglement network is taken to be the inducement of a differential viscoelastic stress. This couples diffusive and mechanical processes through a viscoelastic response where the strain depends upon the amount of penetrant present. Analytically, the major effect is to produce explicit delay terms via a relaxation parameter. This accounts for the fundamental difference between a polymer in its rubbery state and the polymer in its glassy state, namely the finite relaxation time in the glassy state due to slow response to changing conditions. Both numerical and analytical perturbation studies of a boundary value problem for a dry glass polymer exposed to a penetrant solvent are completed. Concentration profiles in good agreement with observations are obtained.
\end{abstract}

Key words. non-Fickian diffusion, polymers, singular perturbation

AMS(MOS) subject classifications. 35F99, 35K20, 35R35, 85A51

1. Introduction. It is clear that polymers and composites are replacing traditional materials in many older scientific, engineering, and commercial applications and that wholly new fields are emerging because of the existence of new synthetic polymers and composites. It is important to have valid theories for their behavior and accurate control of their properties. Central problems emerging in these new technologies are types of diffusive motion quite unlike anything predicted by classical diffusion theory. We shall describe a few of them here.

A major goal of controlled release technology in the pharmaceutical industry is to combine an active agent (the drug or medicine) with its carrier (a polymer) in an economical manner to achieve a release profile that best fits the situation at hand. The resulting devices may be swallowed, smelled, surgically implanted, rubbed on, taped on, or strapped on to selectively reach virtually every part of the body. Many problems or disadvantages associated with conventional usage of pharmaceuticals can be eliminated or greatly alleviated with these new methods; these include systemic effects, patient complicance, and undesirable, inaccurate, fluctuating dosage.

The physical process common to most of controlled release technology is diffusion [1]-[3]. The drugs are released by diffusion alone or by diffusion in combination with other mechanisms. The fabrication and storage of the pharmaceuticals also involve diffusive processes. Many new synthetic polymers are used as separating membranes, encapsulating devices, or impregnated matrices and most of these have intriguing and fascinating diffusive properties [1]-[3].

Many other applications can be mentioned. For example, it is important to understand the mechanisms governing the aging of composites, or mechanisms leading to structural failure of plastics (polymers). Solid rocket propellants are degraded by moisture seepage through polymer encasements. As beverage bottles, polymers serve as barriers to separate gases; as artificial membranes these can be used to allow mixing

* Received by the editors August 8, 1989; accepted for publication (in revised form) April 9, 1990.

$\dagger$ Applied Mathematics 217-50, California Institute of Technology, Pasadena, California 91125. This work was performed under United States Army Research Office (Durham) contract DAAG29-85-K0092, National Science Foundation grant DMS84-00885, Air Force Office of Scientific Research grant AFOSR-870270, and Department of Energy grant W-7405-ENG-36 at the Center for Nonlinear Studies at Los Alamos.

$\ddagger$ Computer Research and Applications, Los Alamos National Laboratory, Los Alamos, New Mexico 87545. This work was performed under United States Department of Energy contract W-7405-ENG-36. 
by controlled diffusion. Polymer masks or templates are used in lithographic techniques for the fabrication of largescale integrated circuits. Diffusion in silicate melts (i.e., highly polymerized liquids) form the basis of the theory of geochemical evolution in magmatic and igneous systems.

All these applications, and many more, involve the diffusive penetration of a glassy polymer by a gas or liquid often accompanied by a phase transition from a glassy state to a rubbery state. For small molecule penetration into a rubbery polymer which remains rubbery, the situation is described by the standard Fickian diffusion equation $C_{t}=\nabla \cdot(D \nabla C)$ in which the diffusivity $D$ is at worst dependent upon the concentration $C$ of the diffusing species, i.e., $D=D(C)$. Although problems involving this equation may be formidable nonlinear boundary value problems, the important qualitative features of diffusion in rubbery polymers can be obtained, and indeed, a rather complete theory exists.

On the other hand, there is no unifying theoretical formulation for glassy polymers. There is not even a complete classification of the various anomalous effects. Experiments and observation indicate that diffusive penetration can depend nonlinearly on changing polymer structure, the state of the system, the previous history of the system, and sometimes on previous rates of change of the system [4]-[7]. Internal stresses can be induced due to differential swelling of different parts of the polymer [8]-[10]. Sharp (shock-like) diffusive fronts may appear; these move with constant speed and are often accompanied by stress and relaxation characteristic of viscoelastic materials [11], [12]. An initially rapid flux may be followed by a drop to a very low value followed by a gradual increase to a steady state [6], [13]. Immobilization (and sometimes reaction) in the interstitial spaces and microvoids can greatly affect the process [14]-[16]. These and many other phenomena have given rise to a partial classification (Case II, Supercase II, Dual mode, Overshoot, Anomalous, non-Fickian,...) of glassy polymers according to various experimental observations.

Because of the relative ease with which they can be obtained experimentally, the quantities most commonly used to define the kinetics of a polymer-penetrant system are $M(t)$, the total amount of penetrant absorbed per unit area of the polymer at time $t$, and $x=s(t)$, the position of the glass-gel (rubber) interface at time $t$. Many diverse observations are reported. The following crude classification has been adopted: Look at $M(t)=k t^{\alpha}$ for short time intervals. Standard Fickian diffusion (i.e., a polymer in its rubbery state above the glass transition temperature) corresponds to the usual $\alpha=\frac{1}{2}$. Case II glassy polymers correspond to $\frac{1}{2}<\alpha<1$, and Super-case II correspond to $\alpha>1$. However, this is not a firm rule, and furthermore, in most glassy polymers $M(t)$ as a function of $t$ does not look like $t^{\alpha}$ for a fixed constant value of $\alpha$; fairly general functions of time are observed [17], [19], some also with their own names (e.g., sigmoid, two-stage, etc.).

These various types of anomalies are often cited as examples of non-Fickian diffusion since they cannot be accounted for by Fickian diffusion theory. The observations are so diverse that rather than any kind of universal theory for glassy polymers, it is probably the case that theories of glassy polymers by still to be defined type will be all that is obtainable. Nevertheless, there are a few common properties which seem to occur in almost all the anomalous observations and the physical implications of these properties can account for the qualitative features of several types of diffusion. For example, Thomas and Windle [12] have proposed that the coupling of diffusive and mechanical properties in terms of a viscoelastic stress field incorporates the dominant physics sufficient to describe the sharp, shock-like concentration front characteristic of Case II diffusion. Neither this type of concentration profile nor its motion 
with constant velocity can arise from Fickian diffusion equation even with a concentration dependent diffusivity.

We adopt and extend the fundamental observation of Thomas and Windle. We derive and study a continuum model resulting from the interaction of diffusion and stress through a viscoelastic response where the stress depends upon the amount of penetrant present. Some type of slow change in deformation involving the polymer's entanglement network is reported in so much of the experimental work that it is not surprising that for properly selected parameter ranges we can account for many of the anomalous cases.

In $\S 2$ we derive our model. We then study our equations in an appropriately chosen range of parameters so that we can produce the sharp fronts characteristic of Case II diffusion and the simultaneous appearance of so-called overshoot. This is done in $\S 3$.

2. The model. The most important and most commonly mentioned property of a glassy polymer is finite relaxation time resulting from the slow response to changing conditions. When a penetrant diffuses into a glassy polymer, a major effect on the polymer's entanglement network is the induction of a differential stress resulting in a concentration front with a slowly varying viscoelastic stress field determined by the polymer's adjustment to the penetrant molecules [11], [12], [19]. Thus diffusive and mechanical processes are coupled through a viscoelastic response where the stress depends upon the amount of penetrant present.

To model this phenomenon we take the total flux $J$ as

$$
J=-D C_{x}-E \sigma_{x}+M C \text {. }
$$

Here $C$ is the concentration of the diffusing penetrant so that $D C_{x}$ represents the diffusive component with the proportionality factor $D$ being the usual diffusivity. Similarly, $\sigma$ is the stress, and $E \sigma_{x}$ is the component of flux representing the viscoelastic effect with the appropriate proportionality factor $E$. Both $D$ and $E$ may depend upon $C$. The term $M C$, with $M$ possibly depending on both $C$ and $\sigma$, represents convective effects which may or may not be present depending upon penetrant molecule size and the size of channels opened in the polymer. Now, we apply the basic equation of continuity (i.e., conservation of mass), namely

$$
C_{t}=-J_{x} \text {. }
$$

Then, (2.1) and (2.2) immediately yield

$$
C_{t}=\left(D C_{x}+E \sigma_{x}\right)_{x}-(M C)_{x} .
$$

Depending upon the geometry of the entanglement network, the size of the penetrant moecules, and many more physical and geometrical properties, polymers can exhibit a wide range of viscoelastic behaviors. If $1 / \beta$ denotes relaxation time and $\eta$ denotes viscosity ( $\beta$ and $\eta$ may both depend on $C$ ), the usual Maxwell model [28] of viscoelasticity is

$$
\sigma_{t}+\frac{\beta}{\eta} \sigma=\beta \varepsilon_{t}
$$

and the usual Voigt model [28] is

$$
\sigma_{t}=\eta \varepsilon_{t}+\beta \varepsilon .
$$

Here $\sigma$ and $\varepsilon$ denote stress and strain, respectively, and the equations result from modelling the solid by springs and dashpots in series or in parallel as shown in Fig. 1. 


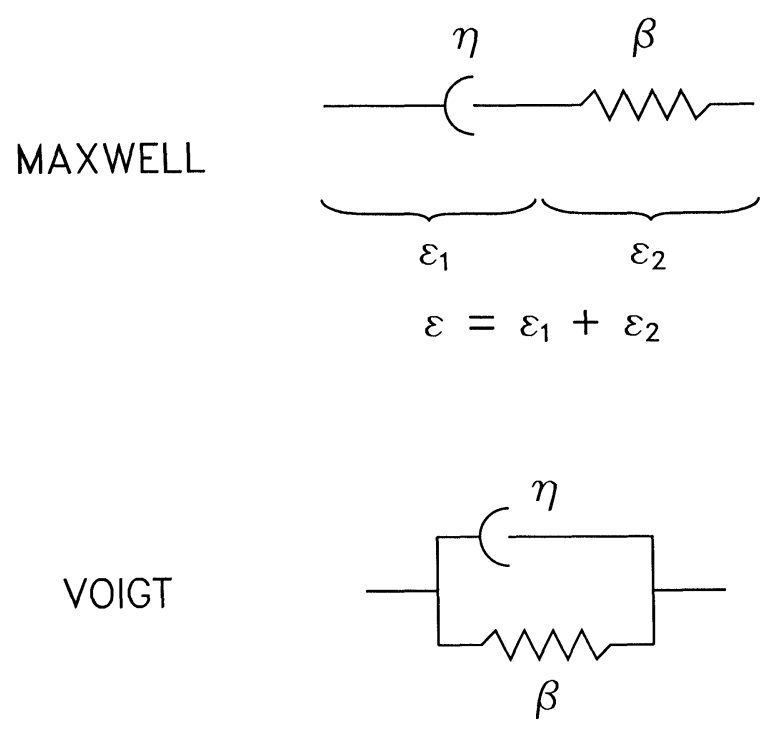

FIG. 1

For solids as complicated as glassy polymers, and in order to be able to account for the rich, diverse behavior observed it is probably better to invoke a generalized model incorporating both (2.4) and (2.5) as special cases. Thus, we shall take as our model

$$
\eta \sigma_{t}+\left(\beta_{1}+\beta_{2}\right) \sigma=\beta_{2}\left(\eta \varepsilon_{t}+\beta_{1} \varepsilon\right),
$$

representing the situation depicted in Fig. 2. Note that limiting cases of (2.6) are the Maxwell model (2.4) when $\beta_{1} \rightarrow 0$ and the Voigt model (2.5) when $\beta_{2} \rightarrow \infty$.

Equations (2.3) and (2.6) involve three unknowns $(C, \sigma$, and $\varepsilon)$. We need a relationship between $\varepsilon$ and $C$ to complete our system, or equivalently a relationship between $\varepsilon$ and $C$ can be used to eliminate $\varepsilon$ leaving two equations in the two unknowns $C$ and $\sigma$. Durning [19] has studied one type of polymer-penetrant system for which he invokes a Maxwell model with $\varepsilon_{t}$ proportional to $C_{t}$. From (2.4) we see that one consequence of this is an equilibrium stress field which is identically zero. There are many common cases, however, [20], where equilibrium or stationary states occur in which there is a residual stress in the glassy part of the polymer or in the neighborhood of the glass-rubber interface. Thus, to derive a model general enough to include many commonly observed situations, we need (2.6) to take the form

$$
\sigma_{t}+\beta \sigma=f\left(C, C_{t}\right)
$$

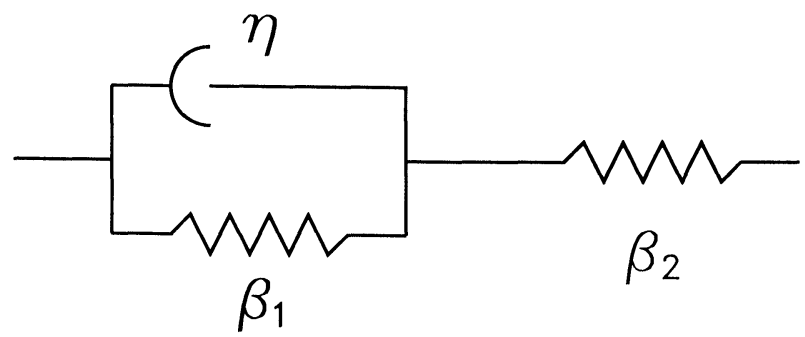

FIG. 2 
Here $\beta=\left(\beta_{1}+\beta_{2} / \eta\right)$, and $f\left(C, C_{t}\right)$ is the model representing the dependence of the stress and/or strain on the concentration field.

Thus, our equations of motion take the form

$$
\begin{gathered}
C_{t}=\left(D C_{x}+E \sigma_{x}\right)_{x}-(M C)_{x}, \\
\sigma_{t}+\beta \sigma=f\left(C, C_{t}\right),
\end{gathered}
$$

subject to appropriate initial and boundary conditions. The various coefficients and nonlinear functions take different forms depending on the particular situation studied. In order to proceed further, we now specialize to a situation representing sharp front formation in a dry, unstressed polymer slab of width $L$, the faces of which are held at constant concentration. Thus,

$$
\begin{gathered}
C(0, t)=C_{0}, \\
C(L, t)=C_{1}>C_{0}, \\
C(x, 0)=0=\sigma(x, 0) .
\end{gathered}
$$

Now, one of two situations occurs depending upon the polymer-penetrant system. Either (i) two distinct regions (glass and rubbery) are formed resulting from a sharp concentration front progressing into the dry glassy polymer and leaving a swollen rubbery polymer behind, or (ii) three distinct regions (glassy, transition, and rubbery) are formed separated by sharp progressing fronts [4], [6], [13]. If $G$ refers to glassy and $R$ to rubbery, then Figs. 3 and 4 illustrate typical forms for the coefficients $\beta$ and $D$ for the two region situation.

For analytical and numerical purposes we shall take

$$
\beta=\beta(C)=\frac{\beta_{G}+\beta_{R}}{2}+\left(\frac{\beta_{R}-\beta_{G}}{2}\right) \tanh \left(\frac{C-C^{*}}{\delta}\right) .
$$

The parameter $\delta$ controls the width of the transition from the glass value $\beta_{G}$ to the rubber value $\beta_{R}$ near the glass transition $C^{*} . D=D(C)$ is easily modelled by an exponential rising from $D_{G}$ and decaying exponentially onto $D_{R}$. For the coefficient $E=E(C)$ the only essential requirement is that $E(0)=0$ to reflect the fact that no

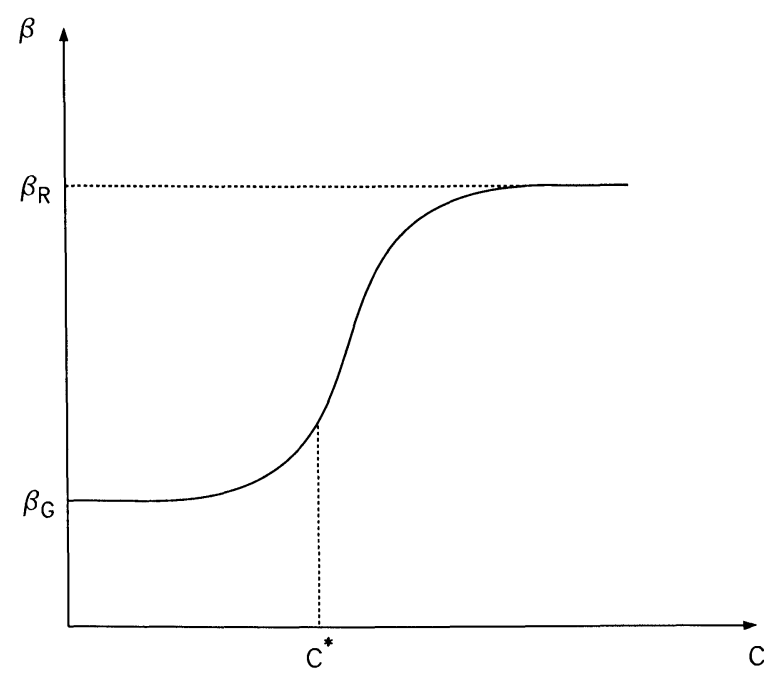

FIG. 3 


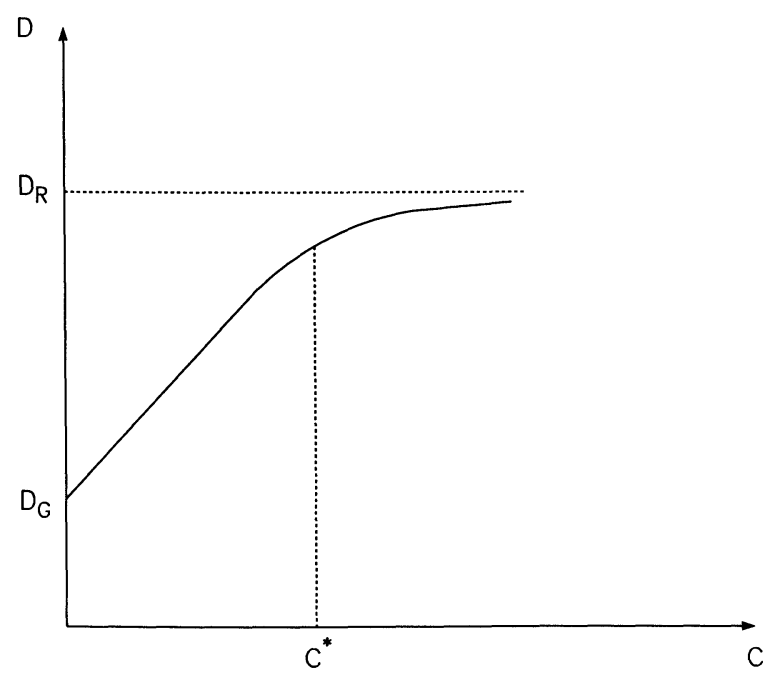

FIG. 4

stress gradient $\sigma_{x}$, however large, can cause a flux of solvent where there is no solvent. An appealing, physically acceptable form for $E(C)$ is $E(C)=\alpha C$ where $\alpha$ is a constant. If $E(0)>0$, then $C(x, t)$ might become negative at some point when a previously established stress gradient continues to force solvent out of a region even after all the solvent is gone. (This has been observed in numerical solutions [21].) For analytical tractability, however, it is convenient to take $E \equiv$ constant $E_{0}$. With respect to accounting for sharp diffusive fronts this form for $E$ is excellent and the deficiency that $E(0)>0$ is no more harmful than the more traditional deficiency of constant coefficient diffusion problems in which we accept infinite signal speed because the characteristics of the parabolic equation are parallel to the time axis.

The physics of the situation suggests that the steady state will look qualitatively something like the profiles sketched in Fig. 5. Thus, we need a function $f\left(C, C_{t}\right)$ such that steady states of (2.9) are consistent with this behavior. An excellent model is given by

$$
f\left(C, C_{t}\right)=\rho(C) C+\nu(C) C_{t},
$$

where $\rho(C)$ and $\nu(C)$ are sketched in Fig. 6. When this is used in (2.9) we see that a steady steady situation implies

$$
\sigma=\frac{\rho(C)}{\beta(C)} C
$$

This is consistent with the behavior sketched in Fig. 5 and yields a zero steady-state stress field in the rubber and a finite nonzero steady-state stress field in the glass. Furthermore, in the transient motion, when the concentration is small (in the glass), the strain is driven by the concentration $C$, and when the concentration is large (in the rubber), the strain is driven by $C_{t}$; this is in agreement with the observations of Durning [19] and Knauss and Kenner [20].

Finally, we shall consider cases in which convective effects are negligible so that $M \equiv 0$. Therefore, (2.8) and (2.9), subject to conditions (2.10)-(2.12), and with the functions $D(C), E(C), M(C, \sigma), \beta(C)$ and $f\left(C, C_{t}\right)$ given as just described, constitute our formulation of the two region problem. In the following sections we solve this 

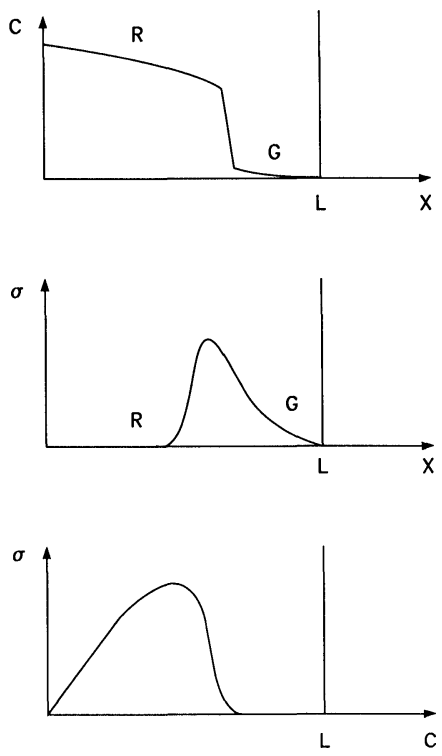

FIG. 5
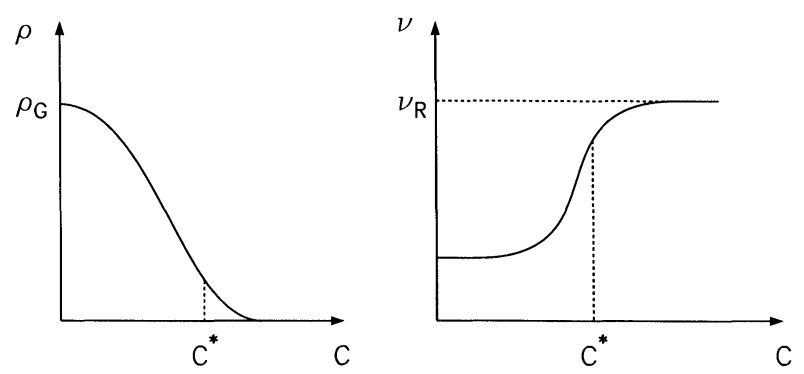

FIG. 6

system. Before we do that, however, we mention some generalizations and consequences of our model.

In those systems where three distinct regions (glassy, transition, and rubbery) are observed with three sets of disparate relaxation times, viscosities, etc., we need make only the obvious modifications in our coefficient functions. For example, we take a $\beta(C)$ like that sketched in Fig. 7. Furthermore, for $f\left(C, C_{t}\right)$ we would simply take $\rho(C)$ and $\nu(C)$ to overlap a little more so that in the transition region $f\left(C, C_{t}\right)$ contains a reasonable amount of both $C$ and $C_{t}$.

Our derivation has been presented for a one-dimensional situation. However, the extension to multidimensions is clear. Furthermore, the same equations of motion will hold in problems having free moving boundaries. Appropriate conditions at the moving boundaries will be needed. These could take many forms; some reasonable ones are discussed in [22]-[24].

Note that $(2.9)$ can be integrated to yield

$$
\sigma(x, t)=\int_{0}^{t} \exp \left[-\int_{\tau}^{t} \beta(C(x, s)) d s\right] f\left(C(x, \tau), C_{t}(x, \tau)\right) d \tau .
$$




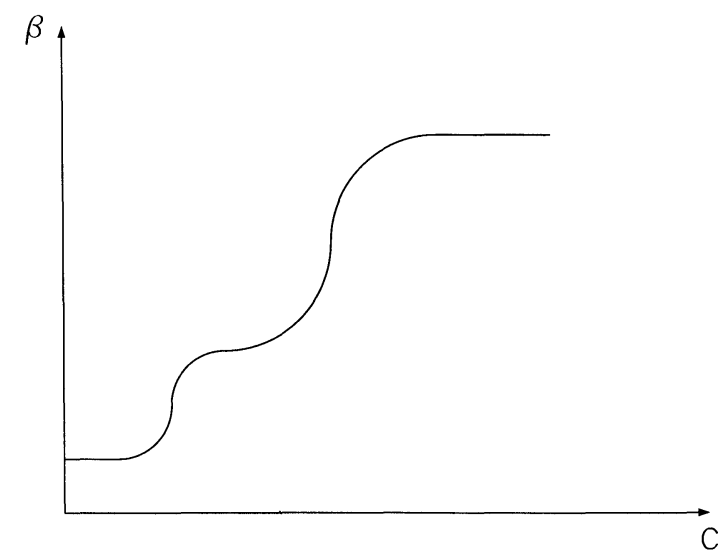

FIG. 7

This result can now be substituted into (2.8) to eliminate all reference to the stress $\sigma$. The resulting integro-differential equation explicitly exhibits the history dependence on past values of $C$ and is of the form postulated by Cohen [25] as being the probable correct analytical formulation for diffusion in glassy polymers. However, for both analytical and numerical methods it is preferable to consider the simultaneous differential (2.8) and (2.9).

We study (2.8) and (2.9) subject to conditions (2.10)-(2.12). We take $D$ and $E$ as constant, $M \equiv 0, \beta(C)$ as given by (2.13), and $f\left(C, C_{t}\right)$ dependent only on $C$ and given by

$$
f\left(C, C_{t}\right)=\rho C \text {. }
$$

We show that this choice retains the dominant mechanisms controlling sharp (shocklike) fronts in the penetrant concentration field; namely, the transition in the instantaneous relaxation time $\beta^{-1}(C)$ at the glass transition $C^{*}$ and the explicit concentration dependence of $f\left(C, C_{t}\right)$. Durning [19] studied diffusion with viscoelasticity given by a Maxwell model like (2.4); that is, with $f\left(C, C_{t}\right)$ a linear function of $C_{t}$ only. With this choice he fails to get sharp front formation. We take the opposite extreme with $f\left(C, C_{t}\right)$ dependent on $C$ only and not on $C_{t}$, and we see that we can account for the observed shock-like fronts.

There are several ways to introduce dimensionless variables. For our purposes it will be convenient to let

$$
\xi=\frac{x}{L}, \quad \tau=\frac{\rho E}{D} t
$$

and

$$
C(x, t)=C_{1} u(\xi, \tau), \quad \sigma(x, t)=\frac{D C_{1}}{E} s(\xi, \tau), \quad \beta\left(C_{1} u\right)=\beta_{R} b(u) .
$$

Then our problem becomes

$$
\begin{gathered}
u_{\tau}=d\left(u_{\xi \xi}+s_{\xi \xi}\right), \quad 0<\xi<1, \quad \tau>0, \\
s_{\tau}=u-\lambda b(u) s, \\
u(0, \tau)=r \\
u(1, \tau)=1, \\
u(\xi, 0)=\sigma(\xi, 0)=0,
\end{gathered}
$$


where

$$
d=\frac{D^{2}}{\rho E L^{2}}, \quad \lambda=\frac{\beta_{R} D}{\rho E}, \quad r=\frac{C_{0}}{C_{1}} .
$$

We now study the problem (2.20)-(2.24).

3. The position and stability of the sharp front. In Cohen and White [26], the normalized differential equations (2.20)-(2.24) are solved numerically. The prevalent features of the solution are (1) initially $\left(\tau<\tau_{0}\right)$, the concentration evolves diffusively; (2) finally $\left(\tau>\tau_{0}\right)$, the concentration relaxes to a steady-state with a discontinuity (as illustrated in Fig. 5) in the limit as $\tau \rightarrow \infty$; and (3) also finally $\left(\tau>\tau_{0}\right)$, the quantity $w=u+s$ is very nearly a straight line with moving end points. We now employ a singular perturbation procedure to show that this is in fact the case. In doing so, we derive a fundamental ordinary differential equation from which we can determine both the (approximate) position and the stability of the sharp front and from which we can reduce the entire problem of placing the sharp front to that of integrating a set of ordinary initial-value problems.

Assume that $0<\varepsilon=(1 / d) \ll 1$. We now construct the asymptotic expansions of the solution of (2.20)-(2.24) as $\varepsilon \rightarrow 0$. We find by the techniques of singular perturbation theory [27] that there is an initial boundary layer of thickness $O(\varepsilon)$ near $\tau=0$ for all $\xi$ in $0 \leqq \xi \leqq 1$. Away from this boundary layer the form of the asymptotic expansion (the outer solution) is given by

$$
u(\xi, \tau, \varepsilon) \sim \sum_{n=0}^{\infty} u_{n}(\xi, \tau) \varepsilon^{n}, \quad s(\xi, \tau, \varepsilon) \sim \sum_{n=0}^{\infty} s_{n}(\xi, \tau) \varepsilon^{n} .
$$

Inserting (3.1) into (2.20)-(2.23), we find by equating like powers of $\varepsilon$ that to first order in $\varepsilon$ we obtain

$$
\begin{gathered}
\frac{\partial^{2} u_{0}}{\partial \xi^{2}}+\frac{\partial^{2} s_{0}}{\partial \xi^{2}}=0, \\
\frac{\partial s_{0}}{\partial t}=u_{0}-\lambda b\left(u_{0}\right) s_{0}, \\
u_{0}(0, \tau)=r, \\
u_{0}(1, \tau)=1 .
\end{gathered}
$$

Note that in obtaining (3.3) we have assumed that $\lambda=O(1)$. The relationship $d=$ $O(1 / \varepsilon) \gg \lambda=O(1)$ implies that

$$
D \gg \beta_{R} L^{2} .
$$

This is a fundamental condition for the validity of the asymptotic arguments of this section.

Equation (3.2) implies that $u_{0}+s_{0}$ is a straight line with varying endpoints; that is, (3.2) implies

$$
u_{0}(\xi, \tau)+s_{0}(\xi, \tau)=A(\tau) \xi+B(\tau)
$$

Upon using the boundary conditions (3.4), (3.5) in (3.7), we find that

$$
\begin{gathered}
A(\tau)=1-r+s(1, \tau)-s(0, \tau), \\
B(\tau)=r+s(0, \tau) .
\end{gathered}
$$


The functions $s(0, \tau)$ and $s(1, \tau)$ are easily determined as follows: evaluate (3.3) at $\xi=0$ to obtain

$$
\frac{\partial s_{0}}{\partial \tau}(0, \tau)=u_{0}(0, \tau)-\lambda b\left(u_{0}(0, \tau)\right) s(0, \tau)=r-\lambda b(r) s(0, \tau) .
$$

This is a simple ordinary differential equation, the solution of which is

$$
s(0, \tau)=\frac{r}{\lambda b(r)}\left[1-e^{-\lambda b(r) \tau}\right] .
$$

Similarly,

$$
s(1, \tau)=\frac{1}{\lambda b(1)}\left[1-e^{-\lambda b(1) \tau}\right]
$$

Therefore, $A(\tau)$ and $B(\tau)$ are explicitly known functions of $\tau$. Finally, we insert (3.7)-(3.9) into (3.3) to obtain

$$
\frac{\partial u_{0}}{\partial \tau}=-u_{0}-\lambda b\left(u_{0}\right) u_{0}+\left[\lambda b\left(u_{0}\right) A(\tau)+A^{\prime}(\tau)\right] \xi+\left[\lambda b\left(u_{0}\right) B(\tau)+B^{\prime}(\tau)\right]
$$

This is an ordinary differential equation in $\tau$ for $u_{0}(\xi, \tau)$ with $\xi$ as a parameter, or in other words, an ordinary differential equation in $\tau$ along each line $\xi=$ constant.

We now obtain the appropriate initial conditions for $u_{0}$ in (3.13) by a standard matching procedure with the asymptotic form (the inner solution) in the initial boundary layer. Set $\tilde{\tau}=\tau / \varepsilon$, and let $u(\xi, \tau)=u(\xi, \varepsilon \tilde{\tau}) \equiv U(\xi, \tilde{\tau}), s(\xi, \tau)=s(\xi, \varepsilon \tilde{\tau}) \equiv S(\xi, \tilde{\tau})$. With this change of variables we find that to first order in $\varepsilon(2.20)-(2.24)$ become

$$
\begin{gathered}
\frac{\partial U}{\partial \tilde{\tau}}=\frac{\partial^{2} U}{\partial \xi^{2}}+\frac{\partial^{2} S}{\partial \xi^{2}}, \\
\frac{\partial S}{\partial \tilde{\tau}}=0, \\
U(0, \tau)=r, \\
U(1, \tau)=1, \\
U(\xi, 0)=S(\xi, 0)=0 .
\end{gathered}
$$

Now, (3.15) and (3.18) imply $S(\xi, \tau) \equiv 0$, so that $U(\xi, \tau)$ evolves purely diffusively and is given by (3.14) with $S \equiv 0$ subject to (3.16)-(3.18). Therefore,

$$
U(\xi, \tilde{\tau})=r+(1-r) \xi+\frac{2}{\pi} \sum_{n=1}^{\infty} \frac{\cos n \pi-r}{n} \exp \left(-n^{2} \pi^{2} \tilde{\tau}\right) \sin n \pi \xi
$$

The standard matching of inner and outer solutions now requires that

$$
\lim _{\tau \rightarrow 0}\left[u_{0}(\xi, \tau)\right]=\lim _{\tilde{\tau} \rightarrow \infty}[U(\xi, \tilde{\tau})]
$$

Therefore,

$$
u_{0}(\xi, 0)=r+(1-r) \xi
$$

and this provides the proper initial condition for (3.13).

Thus, to leading order in $\varepsilon$, the entire problem has been reduced to integrating a set of initial-value problems (3.13) using the initial conditions given in (3.21). Equation (3.13) is of the form

$$
u_{\tau}=F(u, \tau, \xi)
$$


To see how the solution evolves to the steady-state, $\tilde{u}(\xi)$, consider the solution at a fixed instant of time, $\tau=\tau_{1}$. From (3.22), we note that the solution will increase if $F>0$ and decrease if $F<0$. In Fig. 8 we plot a typical neutral curve $F\left(u, \tau_{1}, \xi\right)$ and a typical solution profile. The solution will decrease left of $\xi_{1}$, and increase to the right of $\xi_{1}$, thus tending to form a sharp jump at $\xi_{1}$. However, Fig. 8 illustrates the situation only at $\tau=\tau_{1}$ and in fact the neutral curve $F(u, \tau, \xi)=0$ is time dependent. Thus, the qualitative features are correct, but the processes must be followed dynamically.

One consequence of the structure of (3.22) is the fact that a strict mathematical discontinuity does not develop in finite time. Rather, a steepening front develops and the profile becomes discontinuous only in the limit $\tau \rightarrow \infty$, in the steady state.

In Table 1, we compare this asymptotic estimate of the front position with that obtained in Cohen and White [26] by integrating the full equations (2.20)-(2.24). We took the values $\lambda=1, \beta_{G}=0.1, \beta_{R}=1.0, C^{*}=1 / 2$, and $\delta=0.1$. The asymptotic estimate of the front position was obtained using an Adams-Bashforth-Moulton scheme and bisection to locate the position of the front. We note excellent agreement between the asymptotic position of the front and the actual position, computed by solving the full partial differential equation.

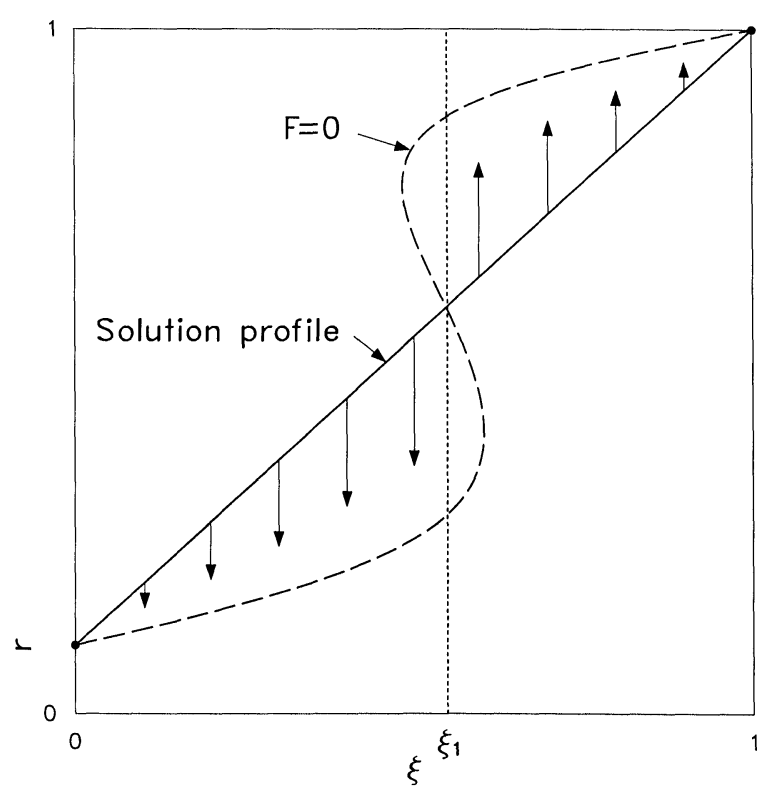

FIG. 8

TABLE 1

\begin{tabular}{ccc}
\hline $\begin{array}{c}\text { Boundary } \\
\text { valve } \\
(r)\end{array}$ & $\begin{array}{c}\text { Asymptotic } \\
\text { position }\end{array}$ & $\begin{array}{c}\text { "Actual" } \\
\text { position }\end{array}$ \\
\hline 0.0 & 0.68855 & 0.688583 \\
0.05 & 0.62584 & 0.624619 \\
0.10 & 0.56346 & 0.560776 \\
0.15 & 0.49126 & 0.486923 \\
0.20 & 0.38512 & 0.384995 \\
\hline
\end{tabular}




\section{REFERENCES}

[1] T. J. Roseman And S. Z. MAnSdorf, EdS., Controlled Release Delivery Systems, Marcel Dekker, New York, 1983.

[2] R. S. SANGer AND D. L. Wise, Eds., Medical Applications of Controlled Release, 2 Vols., CRC Press, Boca Raton, FL, 1984.

[3] R. S. SAnger, Polymeric delivery systems for controlled drug release, Chem. Eng. Commun., 6 (1980), pp. 1-48.

[4] J. CRANK, The Mathematics of Diffusion, 2nd edition, Oxford, 1976.

[5] J. Crank And G. S. Park, Eds., Diffusion in Polymers, Academic Press, New York, 1968.

[6] H. L. FRISCH, Sorption and transport in glassy polymers-a review, Polymer Engr. and Sci., 20 (1980), pp. 2-13.

[7] T. L. SMith AND R. E. ADAM, Effect of tensile deformations on gas transport in glassy polymer films, Polymer, 22 (1981), pp. 299-304.

[8] H. L. Frisch, T. T. WANG, AND T. K. KWEI, Diffusion in glassy polymers, II, J. Polymer Sci., 7 (1969), pp. 879-887.

[9] T. K. Kwei And T. T. WANG, Diffusion in glassy polymers, in Permeability of Plastic Films Coatings, H. B. Hopfenberg, ed., Plenum Press, New York, 1974.

[10] C. H. M. JaCQues, H. B. HopfenberG, AND V. STANnetT, Super-case II transport of organic vapors in glassy polymers, in Permeability of Plastic Films and Coatings, H. B. Hopfenberg, ed., Plenum Press, New York, 1974.

[11] N. Thomas AND A. H. Windle, Transport of methanol in poly-(methyl-methocrylate), Polymer, 19 (1978), pp. 255-265.

[12] N. Thomas And A. H. Windle, A theory of Case II diffusion, Polymer, (1982), pp. 529-542.

[13] C. E. Rogers, Solubility and diffusivity, in Physics and Chemistry of the Organic Solid State, Vol. II, D. Fox, M. M. Sabes, and A. Weissberger, eds., Wiley-Interscience, New York 1965, Chap. 6.

[14] W. R. Vieth And K. J. SladeK, A model for diffusion in a glassy polymer, J. Colloid Sci., 20 (1965), pp. 1014-1033.

[15] J. A. TSHUdy AND C. VON FRAN KENBERG, A model incorporating reversible immobilization for sorption and diffusion in glassy polymers, J. Polymer Sci., 11 (1973), pp. 2027-2037.

[16] D. R. PAUl AND W. J. Kows, Effect of partially immobilizing sorption on permeability and diffusion time lag, J. Polymer Sci., 14 (1976), pp. 675-685.

[17] E. BAGley AND F. A. Song, Two-stage sorption and desorption of organic vapors in cellulose acetate, J. Amer. Chem. Soc., 77 (1955), pp. 2172-2178.

[18] G. Asterita AND S. Joshi, Sample dimension effects in the sorption of solvents in polymersa mathematical model, J. Membrane Sci., 4 (1978), pp. 165-182.

[19] C. J. Durning, Differential sorption in viscoelastic-fluids, J. Polymer Sci., Polymer Phys. Ed., 23 (1985), pp. 1831-1855.

[20] W. G. KNAUSS AND V. H. KENNER, On the hygrothermomechanical characterization of polyvinyl acetate, J. Appl. Phys., 51 (1980), pp. 5131-5136.

[21] R. W. Cox, A Model for stress-driven diffusion in polymers, Ph.D. thesis, California Institute of Technology, 1988.

[22] D. S. COHEN AND C. GoOdhaRT, Sorption of a finite amount of swelling solvent in a glassy polymer, J. Polymer Sci., Part B: Polymer Phys., 25 (1987), pp. 611-617.

[23] D. S. COHEN AND T. ERNEUX, Free boundary problems in controlled release pharmaceuticals. Diffusion in glassy polymers, SIAM J. Appl. Math., 48 (1988), pp. 1451-1465.

[24] - Free boundary problems in controlled release pharmaceuticals. Swelling controlled release, SIAM J. Appl. Math., 48 (1988), pp. 1466-1474.

[25] D. S. Cohen, Theoretical models for diffusion in glassy polymers II, J. Polymer Sci., Polymer Phys Ed., 20 (1984), pp. 1001-1009.

[26] D. S. COHEN AND ANDrEw B. White, Jr., Sharp fronts due to diffusion and stress at the glass transition in polymers, Los Alamos Technical Report 88-2081, June 1988; J. Polymer Sci., to appear.

[27] J. Kevor kian And J. D. Cole, Perturbation Methods in Applied Mathematics, Springer-Verlag, Berlin, New York, 1981.

[28] R. M. Christensen, Theory of Viscoelasticity, Academic Press, New York, 1971. 